\title{
Giant Antiferromagnetically Coupled Moments in a Molecule-Based Magnet with Interpenetrating Lattices
}

\author{
Randy S. Fishman ${ }^{1}$, Satoshi Okamoto ${ }^{1}$, William W. Shum ${ }^{2}$, and Joel S. Miller ${ }^{2}$ \\ ${ }^{1}$ Materials Science and Technology Division, Oak Ridge National Laboratory, Oak Ridge, Tennessee 37831-6071 and \\ ${ }^{2}$ Department of Chemistry, University of Utah, Salt Lake City, Utah 84112-0850
}

(Dated: January 12, 2021)

\begin{abstract}
The molecule-based magnet $\left[\mathrm{Ru}_{2}\left(\mathrm{O}_{2} \mathrm{CMe}\right)_{4}\right]_{3}\left[\mathrm{Cr}(\mathrm{CN})_{6}\right]$ contains two weakly-coupled, interpenetrating sublattices in a body-centered cubic structure. Although the field-dependent magnetization indicates a metamagnetic transition from an antiferromagnet to a paramagnet, the hysteresis loop also exhibits a substantial magnetic remanance and coercive field uncharacteristic of a typical metamagnet. We demonstrate that this material behaves like two giant moments with a weak antiferromagnetic coupling and a large energy barrier between the orientations of each moment. Because the sublattice moments only weakly depend on field in the transition region, the magnetic correlation length can be directly estimated from the magnetization.
\end{abstract}

Due to the importance of weak interactions that are commonly neglected in solid-state materials, moleculebased magnets exhibit a variety of novel behavior [1, 2]. One of the most fascinating and puzzling moleculebased magnets containing two interpenetrating lattices of $\left[\mathrm{Ru}_{2}\left(\mathrm{O}_{2} \mathrm{CMe}\right)_{4}\right]_{3}\left[\mathrm{Cr}(\mathrm{CN})_{6}\right]\left(\mathrm{Me}=\right.$ methyl, $\left.\mathrm{CH}_{3}\right)[3$, , 4] $\left(\mathrm{Cr}\left(\mathrm{Ru}_{2}\right)_{3}\right)$ exhibits an unusual "wasp-waisted" hysteresis loop that is attributed to the weak antiferromagnetic (AF) coupling between its two sublattices [5, 6]. The shape of the initial $M(H)$ data plotted in Fig.1 [7], with a shallow slope followed by a rapid rise, is commonly associated with a metamagnetic transition between $\mathrm{AF}$ and paramagnetic (PM) states [8], which has been observed both in solid-state systems like $\mathrm{FeCl}_{2}[9]$ and DyAl garnet [10] as well as in several other molecule-based magnets 11]. However, typical metamagnets do not exhibit the nonzero remanent magnetization and coercive field found in the hysteresis loop of $\mathrm{Cr}\left(\mathrm{Ru}_{2}\right)_{3}$ compounds with interpenetrating lattices.

We argue that a $\mathrm{Cr}\left(\mathrm{Ru}_{2}\right)_{3}$ compound with interpenetrating lattices behaves like two macroscopic $\mathrm{AF}$ coupled moments with a large energy barrier between the orientations of each moment. Quasi-one- or twodimensional materials that magnetically order in three dimensions due to a weak coupling between sublattices are fairly common, even among molecule-based magnets [12]. Much rarer are materials where each of the weaklyinteracting sublattices is fully ordered in three dimensions.

A single lattice of $\mathrm{Cr}\left(\mathrm{Ru}_{2}\right)_{3}$ is sketched in the inset to Fig.1, where every pair of $\mathrm{Ru}$ ions bridges two $\left[\mathrm{Cr}(\mathrm{CN})_{6}\right]^{3-}(\mathrm{Cr})$ ions located at the corners of the cubic unit cell and separated by $a_{l}=13.4 \AA$. While each $\mathrm{Cr}(\mathrm{III})$ ion has a spin $S=3 / 2$, each $\mathrm{Ru}_{2}$ dimer is in a II/III mixed-valence state with a total spin $S=3 / 2$ [3]. Due to the "paddle-wheel" molecular environment produced by the surrounding four Me groups, each $\mathrm{Ru}_{2}$ moment experiences a strong easy-plane anisotropy with $D \approx 100 \mathrm{~K}$ or $8.6 \mathrm{meV}[13,14]$. The easy plane lies perpendicular to the axis joining the $\mathrm{Ru}_{2}$ sites with the neighboring $\mathrm{Cr}$ ions. Although a single-lattice $\mathrm{Cr}\left(\mathrm{Ru}_{2}\right)_{3}$ compound has been synthesized [5], the resulting samples

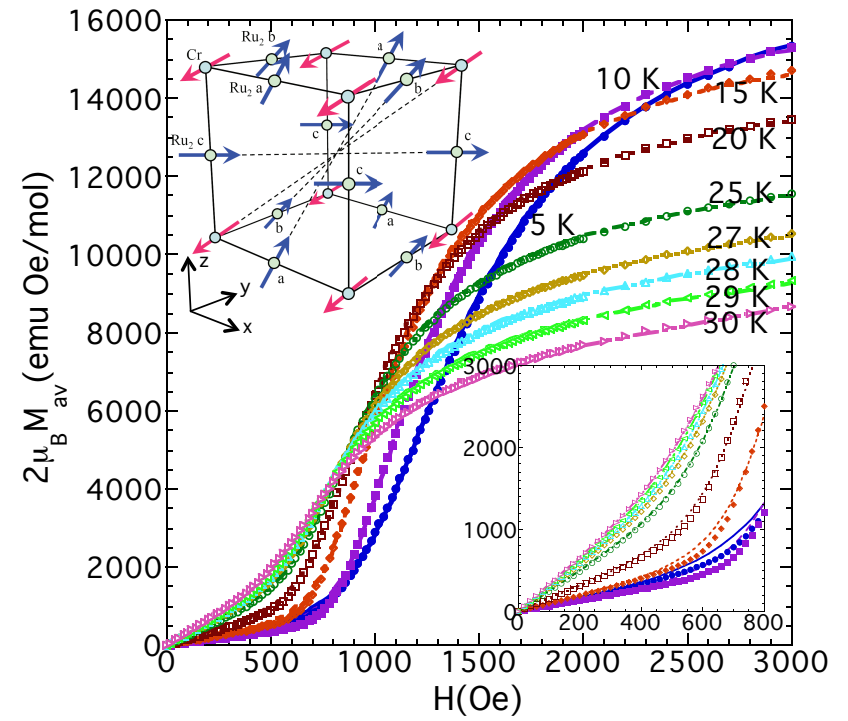

FIG. 1: The initial magnetization (virgin curve) of $\mathrm{Cr}\left(\mathrm{Ru}_{2}\right)_{3}$ with interpenetrating sublattices for temperatures up to 30 $\mathrm{K}$ along with the predicted field dependence. The upper left inset shows the predicted ground state of a single sublattice for classical spins with large anisotropy.

are amorphous. $\mathrm{A} \mathrm{Cr}\left(\mathrm{Ru}_{2}\right)_{3}$ compound with interpenetrating lattices contains a second identical lattice inserted through the open space of the first lattice resulting in a body-centered cubic structure.

Most of the properties of the interpenetrating-lattice compound can be explained by a simple model with strong easy-plane anisotropy $D$ on the $\mathrm{Ru}_{2}$ sites, $\mathrm{AF}$ intra-sublattice exchange $J_{c}$ between neighboring $\mathrm{Cr}$ and $\mathrm{Ru}_{2}$ sites on each sublattice, and a weak $\mathrm{AF}$ intersublattice exchange $K_{c}$ between moments on the two sublattices. The coupling $K_{c}$ is the sum of the dipolar energy and the superexchange interaction through weaklyoverlapping molecular orbitals on the two sublattices [15].

The key to understanding $\mathrm{Cr}\left(\mathrm{Ru}_{2}\right)_{3}$ with interpenetrating lattices is to construct the correct ground state for the single-lattice compound. Each $\mathrm{Ru}_{2}$ spin pair in Fig.1 
is labeled as $a$ (along the $x$ axis), $b$ (along $y$ ), or $c$ (along $z)$. In the classical limit with infinite anisotropy, the $a, b$, or $c$ spins must lie the $y z, x z$, or $x y$ planes, respectively. In the quantum case, the $\mathrm{Ru}_{2}$ spins will have small, but nonzero components in the classically-forbidden directions. For both classical and quantum spins, AF order is frustrated by the easy-plane anisotropy. A similar situation arises in cubic pyrochlores like $\mathrm{Ho}_{2} \mathrm{Ti}_{2} \mathrm{O}_{7}$, where ferromagnetic order is frustrated by local [111] anisotropy [16].

Both classical and quantum calculations provide the same magnetic ground state. The sum of the $\mathrm{Ru}_{2} a, b$, and $c$ spins points opposite to the $\mathrm{Cr}$ spin along one of the four diagonals of the cube. Accounting for the two orientations of the moment along each diagonal, there are eight domains in zero field. For classical spins with infinite anisotropy, the net moment along one of the diagonals is $M_{s l}=(\sqrt{6}-1) S \approx 1.45 S$ per $\mathrm{Cr}$ ion. In both classical and quantum calculations, the $\mathrm{Ru}_{2}$ spins approach the cubic diagonal as $D / J_{c}$ is reduced. The quantum result $M_{s l} \approx 1.21 S$ for the single-lattice moment with $D / J_{c}=5$ is lower than the classical result because the expectation values of the $\mathrm{Ru}_{2}$ spins are reduced in magnitude from 1.5 to about 1.23.

Based on quantum mean-field calculations with $D / J_{c}=5$ and $J_{c}=1.72 \mathrm{meV}$ (see below), the singlelattice compound experiences a spin-flop transition at $H_{s f} \approx 2.78 J_{c} \mu_{B} \approx 80 \mathrm{~T}$ with the field along one of the cubic diagonals. Above $H_{s f}$, the $\mathrm{Cr}$ and total $\mathrm{Ru}_{2}$ moments cant away from the cubic diagonal and, until all the moments become aligned ferromagnetically (FM) at an even higher field, the magnetization $2 \mu_{B} \mathbf{M}$ is no longer parallel to $\mathbf{H}$.

Now consider $\operatorname{Cr}\left(\mathrm{Ru}_{2}\right)_{3}$ with interpenetrating lattices. Assuming that $K_{c} \ll J_{c}$ and $\mu_{B} H \ll J_{c} S$, the sublattice moments $M_{s l} \mathbf{n}_{j}(j=1$ or 2$)$ are rigid with the same spin configuration as in the inset to Fig.1 but pointing along arbitrary cubic diagonals. Thermal equilibrium between the 64 possible configurations $\left\{\mathbf{n}_{1}, \mathbf{n}_{2}\right\}$ is established within correlated clusters of size $\xi$ containing $N_{\mathrm{Cr}} \sim 2\left(\xi / a_{l}\right)^{3} \mathrm{Cr}$ spins per cluster. Although $\xi$ depends on field and may peak in the transition region, the polycrystalline nature of the $\mathrm{Cr}\left(\mathrm{Ru}_{2}\right)_{3}$ samples suggests that the average value of $\xi$ is most important. With $N_{\mathrm{Cr}}$ assumed independent of field, the energy is given by

$$
\begin{aligned}
E & =N_{C r} \sum_{i}\left\{-\mu_{B} M_{s l}\left(\mathbf{n}_{1 i}+\mathbf{n}_{2 i}\right) \cdot \mathbf{H}\right. \\
& \left.+3 K_{c} S^{2} \mathbf{n}_{1 i} \cdot \mathbf{n}_{2 i}\right\}
\end{aligned}
$$

where $\mathbf{H}=H \mathbf{m}$ is the magnetic field and we sum over clusters $i$ The intra-sublattice exchange $J_{c}$ only enters $E$ through the sublattice moments $M_{s l}(T)$, which vanish above $T_{\mathrm{c}} \propto J_{c} S^{2}$.

The zero-field AF ground state is shown schematically in Fig.2(b) with $\mathbf{n}_{1}=-\mathbf{n}_{2}$. Depending on the field orientation, the AF state becomes unstable to one of three possible spin configurations. Configuration III

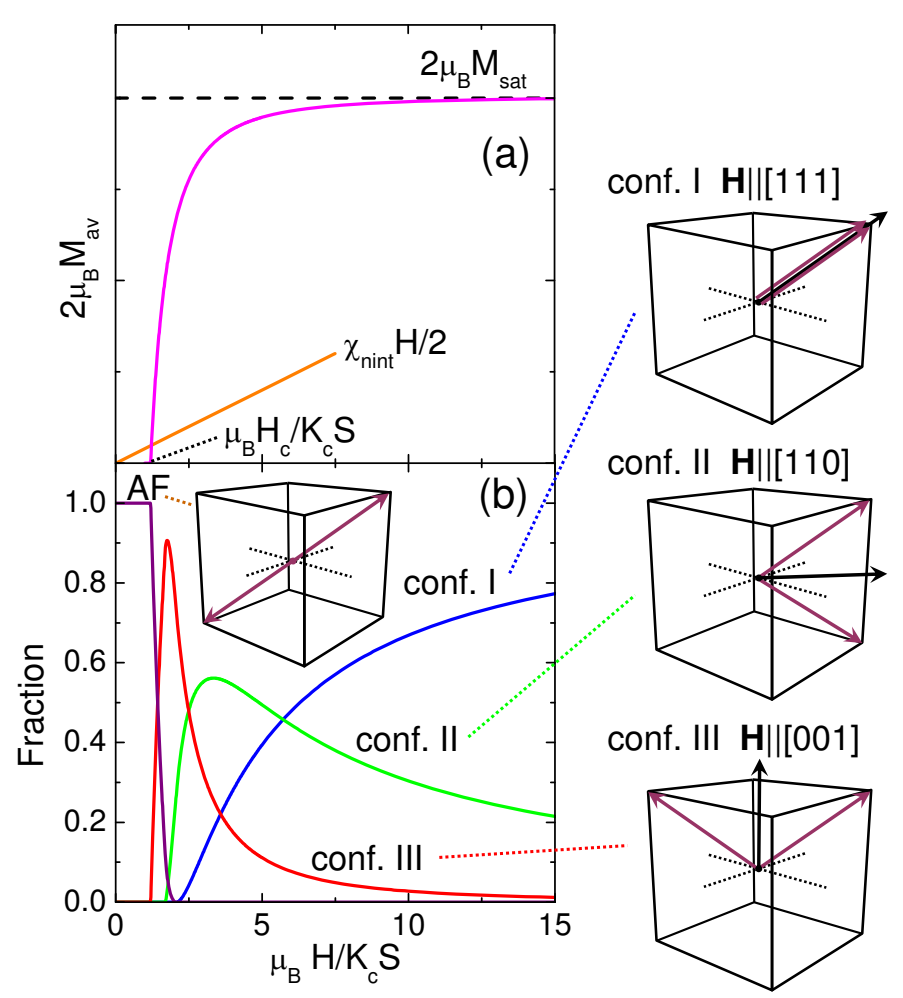

FIG. 2: (a) The $T=0$ average moment and non-interacting linear susceptibility $\chi_{n i n t} H / 2$ and (b) the fractions of the AF state and configurations I, II, and III versus $\mu_{B} H / K_{c} S$ assuming classical spins and large anisotropy.

with $\mathbf{n}_{1} \cdot \mathbf{n}_{2}=-1 / 3$ appears at the critical field $H_{c}=$ $\sqrt{3} K_{c} S^{2} /\left(\mu_{B} M_{s l}\right)$ when $\mathbf{H}$ is parallel to [111]. In configuration II, $\mathbf{n}_{1} \cdot \mathbf{n}_{2}=1 / 3$ and in the PM state labeled configuration I, $\mathbf{n}_{1}=\mathbf{n}_{2}$. The stable phases are shown in Fig.2 along with the fraction of configurations I, II, and III when averaged over all field directions $\mathbf{m}$. Notice that the AF configuration vanishes only with the appearance of the PM state. In zero field, $M_{a v}$ obeys the simple functional form $M_{a v} / M_{s a t}=1-\left(H_{c} / H\right)^{2}$ plotted in Fig.2(a).

We must modify Eq.(1) to account for the small distortion of the single-lattice ground state with field. That distortion is responsible for the small linear susceptibility observed within the AF state at low temperature and small fields, and for the even smaller differential susceptibility $2 \mu_{B} d M / d H$ observed within the PM state at high fields. For classical spins and large anisotropy, the magnetic ground state with $\mathbf{n}_{j}=\mathbf{m}$ cannot be deformed until very large fields, when the $\mathrm{Cr}$ spins tilt away from the $-\mathbf{m}$ direction. But for $\mathbf{n}_{j}=-\mathbf{m}$, the $\mathrm{Ru}_{2}$ spins can easily cant towards the field direction.

So the simplest form for the susceptibility of sublattice $j$ is $\chi_{j}=\chi_{s l} \sin ^{2}\left(\theta_{j} / 2\right)$ where $\theta_{j}=\cos ^{-1}\left(\mathbf{n}_{j} \cdot \mathbf{m}\right)$ is the angle between the field and moment directions. Hence, $\chi_{j}=0$ when $\theta_{j}=0$ and $\chi_{j}=\chi_{s l}$ when $\theta_{j}=\pi$. The non-interacting susceptibility of the magnetic configura- 


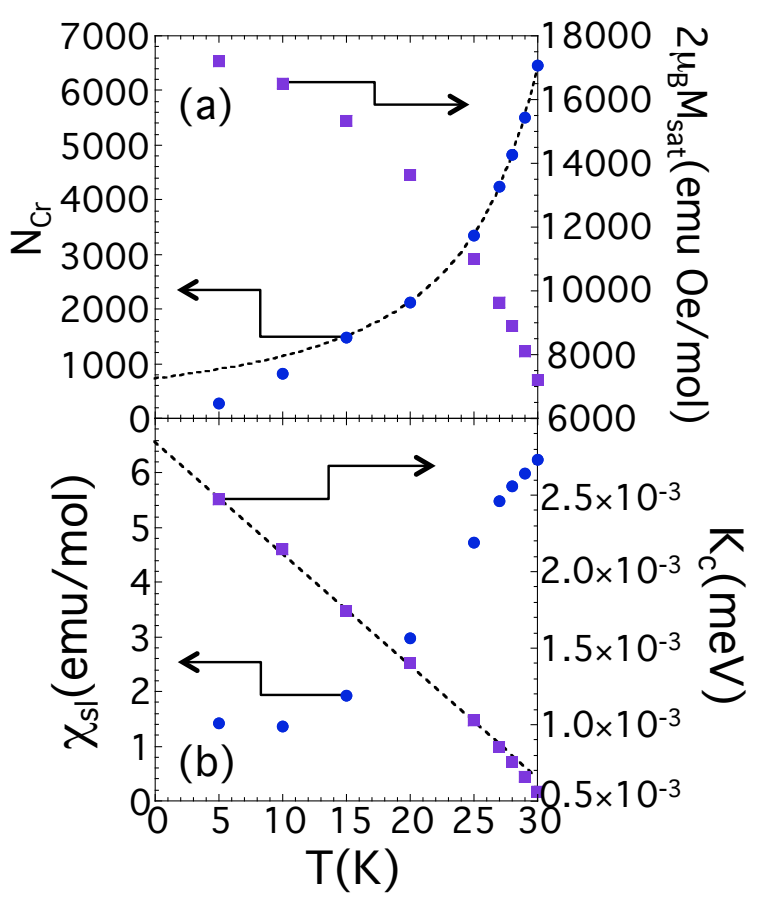

FIG. 3: The fitting parameters versus temperature: (a) the number $N_{\mathrm{Cr}}(T)$ of $\mathrm{Cr}$ atoms in each magnetic cluser (the dashed curve gives the fit $N_{\mathrm{Cr}}=N_{0}\left[\left(T_{\mathrm{c}}-T\right) / T_{\mathrm{c}}\right]^{-3 \nu}$ with $\nu=0.5)$ and the saturation magnetization $2 \mu_{B} M_{\text {sat }}(T)$ and (b) the single-lattice susceptibility $\chi_{s l}(T)$ and the intersublattice interaction $K_{c}(T)$.

tion $\left\{\mathbf{n}_{1}, \mathbf{n}_{2}\right\}$ is then given by $\chi_{\text {nint }}=\chi_{1}+\chi_{2}$ per pair of $\mathrm{Cr}$ atoms and the additional linear term in the magnetization is $N_{\mathrm{Cr}} \chi_{\text {nint }} H / 2$. The extra term $-N_{\mathrm{Cr}} \chi_{n i n t} H^{2} / 4$ must then be added to the energy $E$ of Eq.(1) for each cluster.

In the AF state with $\mathbf{n}_{1}=-\mathbf{n}_{2}, \theta_{2}=\theta_{1}+\pi$ and $\chi_{\text {nint }}=\chi_{s l}$. In the PM state with $\theta_{1}=\theta_{2}, \chi_{\text {nint }}=$ $2 \chi_{s l} \sin ^{2}\left(\theta_{1} / 2\right)$ vanishes when $\theta_{1}=0$ but is nonzero when $\mathbf{H}$ points away from a cubic diagonal. Averaged over all field directions, the susceptibility in the PM phase is $\chi_{\text {nint }} \approx 0.134 \chi_{s l}$. So in agreement with experiments [4, 7], the differential susceptibility at high fields is much smaller than the the linear susceptibility at low fields.

Using this model, we evaluate the partition function by summing over the 64 configurations of $\left\{\mathbf{n}_{1}, \mathbf{n}_{2}\right\}$ and the magnetization by averaging over field directions. For every temperature, the average magnetization depends on four parameters: the single-lattice susceptibility $\chi_{s l}$, the sublattice moment $M_{s l}$, the weak AF interaction $K_{c}$ between sublattices, and the number $N_{\mathrm{Cr}}$ of $\mathrm{Cr}$ atoms within each cluster, half belonging to each sublattice. The fits are provided in Fig.1 and the resulting parameters are plotted in Fig.3.

In Fig.3(a), $N_{\mathrm{Cr}}(T)$ is consistent with the functional form $N_{\mathrm{Cr}}(T)=N_{0}\left[\left(T_{\mathrm{c}}-T\right) / T_{\mathrm{c}}\right]^{-3 \nu}$, where $N_{0} \approx 725$, $T_{\mathrm{c}}=39 \mathrm{~K}$, and $\nu=0.5$, which is the mean-field value for the critical exponent. Since the functional form
$\xi(T) \propto\left[\left(T_{\mathrm{c}}-T\right) / T_{\mathrm{c}}\right]^{-\nu}$ is only expected close to $T_{\mathrm{c}}$, it is remarkable that deviations from this form are only apparent at $10 \mathrm{~K}$. Other evidence for the mean-field nature of the phase transition in $\mathrm{Cr}\left(\mathrm{Ru}_{2}\right)_{3}$ comes from the magnetization of the single-lattice compound [4], which although amorphous closely follows a Brillouin function and vanishes near $T_{\mathrm{c}}$ like $\left(T_{\mathrm{c}}-T\right)^{1 / 2}$.

The "saturation" magnetization $2 \mu_{B} M_{\text {sat }}(T)=$ $\sqrt{3} \mu_{B} M_{s l}(T)$ per Cr atom plotted in Fig.3(a) gives the average magnetization when the two nondistorted sublattice moments are aligned. Nevertheless, the magnetization continues to rise with increasing field due to the deformation of the ferrimagnetic ground state of each sublattice. Indeed, Vos et al. [4] found that the magnetization at $5 \mathrm{~K}$ rises from $16,937 \mathrm{emu} \mathrm{Oe} / \mathrm{mol}$ at 0.5 $\mathrm{T}$ to $20,800 \mathrm{emu} \mathrm{Oe} / \mathrm{mol}$ at $5 \mathrm{~T}$, far above the "saturation" value of $17,286 \mathrm{emu} \mathrm{Oe} / \mathrm{mol}$. In rough agreement with the assumed form for the non-interacting susceptibility $\chi_{n i n t}$, the high-field differential susceptibility of $0.086 \mathrm{emu} / \mathrm{mol}$ is about nine times smaller than the low-field linear susceptibility of $0.81 \mathrm{emu} / \mathrm{mol}$. Notice that the $30 \mathrm{~K}$ clusters with $N_{\mathrm{Cr}}=6464$ have correlation lengths $\xi \sim 15 a_{l}=200 \AA$ and sublattice magnetizations $\mu_{B} N_{\mathrm{Cr}} M_{s l}(T=30 \mathrm{~K}) \approx 4500 \mu_{B}$.

At $T=5 \mathrm{~K}$, the saturation magnetization is significantly lower than the classical result with $D=\infty$. But the single-lattice moment $M_{s l}$ for quantum spins decreases rapidly with increasing $D / J_{c}$ from its maximal value of $2 S$ per $\mathrm{Cr}$ ion when $D=0$. The fitted saturation value can be used to estimate that $D / J_{c} \approx 5$ and $J_{c} \approx 1.72 \mathrm{meV}$. Another estimate for $J_{c}$ comes from the mean-field result $T_{\mathrm{c}} \approx 4.05 J_{c}=33 \mathrm{~K}$ for the transition temperature with $D / J_{c}=5$, yielding the smaller value $J_{c} \approx 0.70 \mathrm{meV}$. However, mean-field theory typically overestimates $T_{\mathrm{c}}$ and underestimates $J_{c}$. So at low temperatures, the inter-sublattice coupling $K_{c}$ is roughly 600 times smaller than the intra-sublattice coupling $J_{c}$.

Plotted in Fig.3(b), the sublattice susceptibility $\chi_{s l}$ rises sharply with increasing temperature and is roughly proportional to $N_{\mathrm{Cr}}$. The inter-sublattice coupling $K_{c}(T)$ falls off almost linearly with temperature below $25 \mathrm{~K}$. If $K_{c}$ were short ranged and coupled sublattice moments at distinct points in space, we would expect that $K_{c}(T) \propto M_{s l}(T)^{2}$. The temperature dependence of $K_{c}(T) / M_{s l}(T)^{2}$ may be ascribed to the complex and long-ranged interaction between the two sublattices, each of which contains two species of magnetic ions with different temperature-dependent average moments.

Three of the four fitting parameters in this model, $M_{s l}(T), N_{\mathrm{Cr}}(T)$, and $\chi_{s l}(T)$, are properties of the singlelattice compound; only $K_{c}(T)$ reflects the presence of two sublattices. Above about $30 \mathrm{~K}$, the fits break down primarily because the linear terms $\chi_{s l}(T) \sin ^{2}(\theta / 2) H$ in the sublattice magnetization are no longer small compared to the zero-field value of $2 \mu_{B} M_{s l}(T)$. Consequently, the inter-sublattice coupling $K_{c}$ effectively depends on field as well as on temperature.

Despite the success of the fits below $30 \mathrm{~K}$, the lower- 
right inset to Fig.1 suggests one limitation of our model. Although the correlation length $\xi$ must reach a maximum near $H_{c}(T)=\sqrt{3} K_{c}(T) S^{2} /\left(\mu_{B} M_{s l}(T)\right)$ as fluctuations soften, our model assumes that $\xi(T)$ is independent of field. Consequently, the curves in Fig.1 lie slightly above the data points near $H_{c}(T)$, especially at lower temperatures. A correlation length $\xi(T, H)$ that peaks in the vicinity of $H_{c}(T)$ would produce even better agreement with the experimental data. Because the thermal averages are assumed to be independent from one cluster to another, the model magnetization is a continuous function of $H$ for any nonzero temperature and for any orientation of the magnetic field. Due to the polycrystalline sample, we cannot say whether the experimental magnetization of $\mathrm{Cr}\left(\mathrm{Ru}_{2}\right)_{3}$ experiences a jump near $H_{c}(T)$ at nonzero temperatures, as observed in single crystals of conventional metamagnets [9, 10] up to about $85 \%$ of the Néel temperature.

The interpenetrating-lattice compound $\mathrm{Cr}\left(\mathrm{Ru}_{2}\right)_{3}$ may be the only known material with two or more weaklyinteracting three-dimensional sublattices. Another molecule-based magnet that bears some similarities to $\mathrm{Cr}\left(\mathrm{Ru}_{2}\right)_{3}$ is methylamine chrome alums [17], which contains $\mathrm{Cr}$ (III) ions in a face-centered cubic structure. Although weak AF dipolar interactions couple the four sublattice moments, the intra-sublattice exchange is negligible in chrome alums. So the three-dimensional ordering at about $0.02 \mathrm{~K}$ is also produced by the dipolar interactions between magnetic sublattices.

Most of the puzzling properties of $\mathrm{Cr}\left(\mathrm{Ru}_{2}\right)_{3}$ with interpenetrating lattices can be explained by two macro- scopic moments that are weakly AF coupled with a large energy barrier between the orientations of each moment. If the individual moments within each sublattice rotate together, then the energy barrier between moments in the [111] and [111] directions for classical spins at $T=0$ is $\Delta(0)=0.07 J_{c} S^{2} N_{\mathrm{Cr}}$. At nonzero temperatures, we expect that $\Delta(T) \propto N_{\mathrm{Cr}}(T) M_{s l}(T)^{2} J_{c} \sim$ $N_{0} J_{c}\left[\left(T_{\mathrm{c}}-T\right) / T_{\mathrm{c}}\right]^{-1 / 2}$. So $\Delta(T)$ will rise very close to $T_{c}$, which may explain the anomalous AC susceptibility [3] observed in these materials.

Our work provides several new predictions for $\mathrm{Cr}\left(\mathrm{Ru}_{2}\right)_{3}$ with interpenetrating lattices. In the magnetic ground state, the total moment of each sublattice must lie along one of the four cubic diagonals. This can be verified by neutron scattering, either on a deuterated sample or with a large enough polycrystal. With a single crystal, the transitions between the AF and configurations I, II, or III should be observable for different orientations of the field. The predicted correlation length $\xi(T)$ can be verified independently by fitting the elastic peaks measured with neutron scattering, even on a polycrystalline sample. We hope that this paper will inspire future work on this fascinating system, including measurements on single crystals when they become available.

Acknowledgements. We would like to acknowledge useful conversations with Prof. Bruce Gaulin. This research was sponsored by the Division of Materials Science and Engineering of the U.S. Department of Energy and by the U.S. National Science Foundation (Grant No. 0553573).
[1] J.S. Miller and A.J. Epstein, Angew. Chem. internat. edit. 33, 385 (1994); J.S. Miller and A.J. Epstein, $M R S$ Bull. November, 21 (2000); J.S. Miller, Adv. Mat. 14, 1105 (2002).

[2] S.J. Blundell and F.L. Pratt, J. Phys.: Cond. Mat. 16, R771 (2004).

[3] Y. Liao, W.W. Shum, and J.S. Miller, J. Am. Chem. Soc. 124, 9336 (2002).

[4] T.E. Vos, Y. Liao, W.W. Shum, J.-H. Her, P.W. Stephens, W.M. Reiff, and J.S. Miller, J. Am. Chem. Soc. 126, 11630 (2004).

[5] T.E. Vos and J.S. Miller, Angew. Chem. 44, 2416 (2005).

[6] J.S. Miller, T.E. Vos, and W.W. Shum, Adv. Mat. 17, 2251 (2005).

[7] W.W. Shum, J.N. Schaller, and J.S. Miller, J. Phys. Chem. C 112, 7936 (2008).

[8] E. Stryjewski and N. Giordano, Adv. Phys. 26, 487 (1977).

[9] I.S. Jacobs, and P.E. Lawrence, Phys. Rev. 164, 866 (1967); P. Wong, P.M. Horn, R.J. Birgeneau, and G. Shirane, Phys. Rev. B 27, 428 (1983).

[10] D.P. Landau, B.E. Keen, B. Schneider, and W.P. Wolf, Phys. Rev. B 3, 2310 (1971).

[11] M. Ohba, H. Kawa, N. Fukita, and Y. Hashimoto, J. Am. Chem. Soc. 119, 1011 (1997); M.K. Saha, M.C. Morón, F. Palacio, and I. Bernal, Inorg. Chem. 44, 1354 (2005);
M.L. Taliaferro, F. Palacio, and J.S. Miller, J. Mater. Chem. 16, 2677 (2006).

[12] E. Coronado, J.R. Galán-Mascarós, C.J. Gómez-García, and C. Martí-Gastaldo, Inorg. Chem. 44, 6197 (2005).

[13] V.M. Miskowiski, M.D. Hopkins, J.R. Winkler, and H.B. Gray in Inorganic Electronic Structure and Spectroscopy, Vol. 2, edited by E.I. Solomon and A.B.P. Lever (John Wiley \& Sons, New York, 1999), Ch. 6.

[14] W.W. Shum, Y. Liao, and J.S. Miller, J. Phys. Chem. A 108, 7460 (2004).

[15] For easy-plane anisotropy, the $\mathrm{Cr}-\mathrm{Cr}$ dipolar interactions between sublattices cancel by symmetry while the Cr$\mathrm{Ru}_{2}$ and $\mathrm{Ru}_{2}-\mathrm{Ru}_{2}$ dipolar interactions produce intersublattice interactions that are AF and FM, respectively, with a net FM dipolar interaction between sublattices. At low temperatures, the observed AF interaction $K_{c}$ can be recovered with an additional inter-sublattice superexchange between neighboring $\mathrm{Ru}_{2}$ pairs separated by $a_{l} / 2$ of $3.1 \times 10^{-3} \mathrm{meV}$, using the sublattice ground state provided by a quantum calculation with $D / J_{c}=5$.

[16] M.J. Harris, S.T. Bramwell, D.F. McMorrow, T. Zeiske, and K.W. Godfrey, Phys. Rev. Lett. 79, 2554 (1997).

[17] M.C.M. O'Brien, Phys. Rev. 104, 1573 (1956); K. Motizuki, J. Phys. Soc. Japan 14, 759 (1959). 